\title{
Prevalencia de Cryptosporidium spp. y Giardia spp. en terneros, y su presencia en agua y en niños con problemas digestivos en el cantón San Fernando, Ecuador
}

\section{Teófilo Estuardo Palacios Ordóñez}

Facultad de Ciencias Agropecuarias de la Universidad de Cuenca, Cuenca, Ecuador. Autor para correspondencia: estuardo.palacios@ucuenca.edu.ec

Fecha de recepción: 3 de agosto 2016 - Fecha de aceptación: 15 de marzo 2017

\section{RESUMEN}

Objetivo: Determinar la prevalencia de Cryptosporidium spp. y Giardia spp. en heces de terneros de 04 meses de edad, como factor contaminante de los recursos hídricos de uso de la población humana de 2-6 años de edad, en el cantón San Fernando, provincia del Azuay.

Metodología: Es un estudio epidemiológico de corte transversal-descriptivo, realizado durante el período septiembre del 2013 a abril del 2014. Se utilizó una muestra de 120 terneros de 0 a 4 meses de edad, que de acuerdo a los datos de AGROCALIDAD es la totalidad de terneros del cantón; 42 niños de 2 a 6 años de edad con problemas digestivos en su visita al centro de salud y 40 litros de agua obtenida de la zona de captación en el sector del Hato de la Virgen. En terneros y niños se utilizó materia fecal que fue tratada para realizar la coloración de Ziehl-Neelsen para Cryptosporidium spp. y la técnica de Ritchie para Giardia spp. En el agua se determinó la cantidad de quistes y ooquistes /100 ml por el método EPA 1623. Los niños fueron discriminados para el estudio según el origen (rural o urbano). Se aplicó una estadística descriptiva con la ayuda del software SPSS versión 22.

Resultados: Se determinó que en terneros existe una prevalencia del $93.3 \%$ de Cryptosporidium spp., (112 casos positivos) y de 76.7\% de Giardia spp. (92 casos positivos). En agua, se determinó la presencia de 5 ooquistes de Cryptosporidium spp./100 ml y 10 quistes de Giardia spp./100 ml. La prevalencia de Cryptosporidium spp. en los niños alcanzó una media del 14.3\%, mientras que la de Giardia spp. fue del $33.3 \%$.

Conclusiones: El estudio demostró una alta prevalencia de Cryptosporidium spp. y Giardia spp. en los terneros, quienes serían en buena medida los responsables de la alta concentración en agua y de la alta prevalencia en niños con problemas digestivos en el cantón San Fernando.

Palabras clave: Cryptosporidium spp., Giardia spp., prevalencia, terneros, ooquistes y quistes en agua, problemas digestivos en niños, cantón San Fernando.

\footnotetext{
ABSTRACT

Objective: To determine the prevalence of Cryptosporidium spp. and Giardia spp. in the feces of calves 0-4 months of age, as contaminating factor of the water resources used by the human population of 2-6 years old, in the canton San Fernando, province of Azuay.

Methodology: This is a cross-sectional epidemiological study carried out during the period from September 2013 to April 2014. A sample of 120 calves from 0 to 4 months of age was used, according to AGROCALIDAD data; 42 children from 2 to 6 years of age with digestive problems in their visit to the health center; and 40 liters of water obtained from the catchment area in the Hato de la Virgen sector. In calves and infants, fecal material was collected and treated to perform the Ziehl-Neelsen coloration for Cryptosporidium spp. and the Ritchie technique for Giardia spp.; in the water, the number of cysts and oocysts $/ 100 \mathrm{ml}$ were determined by the EPA method 1623 . The children were discriminated for the study according to the origin (rural or urban). Descriptive statistics were applied with the SPSS 22.
} 
Results: A prevalence of 93.3\% Cryptosporidium spp. (112 positive cases) and 76.7\% Giardia spp. (92 positive cases) was found in calves. In water, the presence of 5 oocysts of Cryptosporidium spp. $/ 100 \mathrm{ml}$ and 10 cysts of Giardia spp./100 ml. The prevalence of Cryptosporidium spp. in children reached an average of $14.3 \%$, while that of Giardia spp. was $33.3 \%$.

Conclusions: The study demonstrated a high prevalence of Cryptosporidium spp. and Giardia spp. in calves, who would be largely responsible for the high concentration in water and high prevalence in children with digestive problems in the canton San Fernando.

Keywords: Cryptosporidium spp., Giardia spp., prevalence, calves, oocysts and cysts in water, digestive problems in children, San Fernando canton.

\section{INTRODUCCIÓN}

Las enfermedades parasitarias intestinales son las más recurrentes en todo el mundo, especialmente en los grupos pobres de los países en vías de desarrollo. Se estima que 3,500 millones de personas están afectadas por estas infecciones, siendo en su gran mayoría niños (Tedesco et al., 2012). La gran parte de parásitos intestinales son transmitidos al ser humano por la contaminación del ambiente, por lo que el agua y los alimentos están expuestos a esta problemática insalubre (Solarte et al., 2010). Se estima que el $4 \%$ del total de muertes en el mundo se debe a factores relacionados con el agua, desagües e higiene (Medina et al., 2012).

Las parasitosis intestinales pueden ser producidas por dos grupos de organismos: protozoarios y helmintos. Entre los protozoarios más comunes se encuentran Giardia lamblia y Cryptosporidium spp. (Tedesco et al., 2012). Cryptosporidium spp. y Giardias spp. son parásitos intestinales que pueden ocasionar diarrea, náuseas, calambres y otros síntomas; en su etapa contagiosa viven en formas microscópicas que se conocen como ooquistes y quistes respectivamente, que se pueden encontrar en desperdicios sanitarios de personas o animales infectados. Las lluvias y las aguas negras pueden llevar estos desperdicios sanitarios a los ríos, quebradas, lagos, etc., y así contaminar las fuentes de agua potable (Arizona Department of Environmental Quality, 1995).

En un estudio realizado en Colombia para determinar la incidencia de este parásito en niños con cáncer, Carreño et al. (2005) concluyeron que el protozoario Cryptosporidium puede intervenir en el sistema inmunológico de estos pacientes, evidenciándose en la materia fecal y la deshidratación. De igual manera, De Arango et al. (2006) señalan que en el 42\% de niños con complicaciones del sistema inmune por cáncer en Colombia, se evidencia la prevalencia de este parásito en su materia fecal. Del Coco et al. (2009) indican que el Cryptosporidium es uno de los parásitos más comunes en terneros, lo cual ha sido bastante estudiado en muchos países. Estos autores estudiaron a grupos de terneros en diferentes localidades de Argentina, detectando que este parásito es una de las causas de diarrea neonatal bovina, con una prevalencia del 17\%. Un estudio similar realizado por Modini et al. (2010) comprobaron que el $84 \%$ de terneros de la cuenca Lechera Santafesina (Argentina) resultó ser positivo a los análisis de Cryptosporodium spp.

Algunos estudios realizados en países de América Latina señalan que el 12\% de la población infantil presenta altas tasas de prevalencia de Giardia spp. (Lozano et al., 2009). Así mismo, en un estudio realizado a un grupo de niños de escasos recursos económicos que asisten al Templo Comedor Sagrado Corazón Teresa Benedicta de la Cruz de Medellín (Colombia), dichos autores encontraron una prevalencia del 58\% del mencionado parásito. Para Giraldo et al. (2005), las infecciones causadas por Giardia lamblia tienen mayor incidencia en lugares tropicales y subtropicales y tienen más prevalencia en niños y adultos mayores, impidiendo el crecimiento y el desarrollo del sistema inmunológico. Estos autores realizaron un estudio en niños que asisten a hospitales del Instituto Colombiano de Bienestar Familiar en Armenia, determinando que en el $12.8 \%$ de niños, el protozoario más frecuente es Giardia lamblia.

En términos generales, ambos parásitos mantienen características similares de contagio y relación con la insalubridad. Es así que la Agency Environmental Protection (2012) ratifica que la propagación de estos protozoarios está estrechamente relacionada con los productos lecheros, lo que hace relevante 
y que sea de mucho interés el presente artículo. En razón de sus grandes potenciales patogénicos, Cryptosporidium spp. y Giardia lamblia también están incluidos en la lista de contaminantes microbianos del agua potable.

La forma de transmisión de estos protozoarios para convertirse en una zoonosis, es por la ingestión de alimentos y agua que se encuentran contaminadas con material fecal de individuos infectados, el contacto directo con heces infectadas o la ingestión de agua de lagunas, quebradas o ríos contaminados con efluentes de alcantarillado o de granjas para cría de bovinos (Acha \& Szyfres, 2003).

Los objetivos del presente estudio fueron: 1) determinar la prevalencia de Cryptosporidium spp. y Giardia spp. en las heces de terneros de 0-4 meses de edad; 2) evaluar la cantidad de ooquiste y quistes de estos parásitos en el agua utilizada en la región; y 3) evaluar la presencia de ooquiste y quistes de los protozoarios en materia fecal de niños padeciendo diarreas en el cantón San Fernando, provincia del Azuay.

\section{MATERIALES Y MÉTODOS}

De acuerdo al número de veces que se estudiaron las unidades muéstrales, el presente estudio epidemiológico fue de carácter observacional; fue transversal y se considera prospectivo pues no existen mediciones previas de estas variables en las tres unidades de estudio. Este estudio presenta los valores de la prevalencia de Cryptosporidium spp. y Giardia spp. en tres unidades distintas: terneros de 0-4 meses de edad, agua de los tanques de captación para el sistema de potabilización y riego, y niños de 26 años de edad. En estos últimos se realizó un análisis explicativo para determinar la posible asociación entre la procedencia y la presencia de estos parásitos en los niños.

La población de terneros, entre 0 y 4 meses de edad en el cantón en estudio, fue de 120 animales, (según los controles de vigilancia y seguridad alimentaria de Agrocalidad-Azuay, con respecto a la fase de vacunación contra la Fiebre Aftosa). Para los fines de este estudio se decidió abarcar a todos los terneros de esta edad. Las muestras de materia fecal de los terneros fueron tomadas directamente de la ampolla rectal de cada uno de ellos y por una sola vez, tomando en consideración que el $50 \%$ de terneros presentaban cuadros de diarrea sin causa específica. Éstas fueron recolectadas en recipientes plásticos y estériles en una cantidad aproximada de $40 \mathrm{~g}$; luego fueron trasladadas al Laboratorio Clínico Veterinario de la Escuela de Medicina Veterinaria y Zootecnia de la Universidad de Cuenca, en donde se procedió a realizar el estudio microscópico, utilizando las técnicas de coloración de Ziehl-Neelsen para la observación de ooquistes de Criptosporidium spp. y la técnica de Ritchie para observar quistes de Giardias spp., procesadas de la siguiente manera:

- Técnica de coloración de Ziehl-Neelsen: se procedió a realizar el extendido de la muestra en un portaobjetos y se dejó secar al ambiente para luego fijar con metanol por 10 minutos;

posteriormente se agregó el colorante de Carbol fucsina y se dejó actuar por 20 minutos. Se lavó con agua corriente y se decoloró con ácido sulfúrico al 7\%. Como colorante de contraste se utilizó verde de Malaquita por el tiempo de 2 minutos para finalmente proceder al lavado, secado y observación al microscopio con el lente 40X.

- Técnica de Ritchie: la muestra se diluyó con solución fisiológica isotónica en cantidad aproximada de $10 \mathrm{ml}$, se filtró para eliminar restos de ingesta y se procedió a centrifugar por 2 minutos y decantar el sobrenadante; se homogenizó el sedimento y se adicionó $10 \mathrm{ml}$ de formol al 10\%, se mezcló y se dejó reposar por 5 minutos, luego se agregó $3 \mathrm{ml}$ de éter, se tapó y se mezcló fuertemente para proceder a centrifugar por el tiempo de 2 minutos a $1500 \mathrm{rpm}$, posteriormente se eliminó las tres capas formadas, quedando solamente el sedimento y una gota de éste se colocó en el portaobjetos con una gota de lugol, finalmente se colocó un cubreobjetos y se observó en microscopio con el lente 40X.

La población infantil comprendida entre 0-9 años en el cantón San Fernando según el CENSO del 2010 fue de 673 niños. Debido a que no se tuvo acceso directo a las personas de esta población, se decidió, de común acuerdo con el Centro de Salud del cantón, realizar un muestreo consecutivo bajo el criterio de inclusión de todos los sujetos, del rango de edad de 2 a 6 años, que acudieron al Centro de 
Salud en el periodo comprendido entre noviembre del 2013 - abril del 2014. El número de niños entre 2 a 6 años de edad que acudieron al Centro de Salud fue de 87, de los cuales solamente 42 presentaron problemas digestivos. Estos 42 niños constituyeron la muestra a la que se aplicó el estudio. Por lo tanto, se trató de una muestra no probabilística discrecional pues los sujetos de estudio fueron elegidos para constatar un objetivo específico relacionado con los problemas digestivos, descartando aquellos que no presentaron problemas digestivos.

Las muestras de agua se tomaron en el tanque de captación en el sector del Hato de la Virgen, que distribuye este líquido con tres propósitos: 1) consumo humano, urbano-potable y rural-no-potable; 2) sistema de riego; y 3) abrevaderos de animales. El agua se colectó en dos recipientes estériles con capacidad de 20 litros cada uno que se colocaron en contacto con sus respectivas fundas de hielo y geles refrigerantes y fueron llevados al laboratorio de EPMAPS en la ciudad de Quito, con las respectivas seguridades de protección en la conservación y preservación necesarias para el análisis.

Para la determinación de ooquistes y quistes se utilizó el método EPA 1623, que se fundamenta en un método inmunológico de reacción específica antígeno anticuerpo. Se utilizaron los reactivos anti crypto y anti giardia, los cuales son anticuerpos monoclonales específicos, es decir que la adición de este reactivo permitió hacer un complejo antígeno crypto o giardia presente en la muestra, más anticuerpo marcado, que se produce mediante rotación a $19 \mathrm{rpm}$ durante una hora. Luego se evidenció la presencia de ooquistes y quistes mediante una tinción deisotiocianato de fluoresceína en el microscopio a través de una luz fluorescente.

Se aplicó una estadística descriptiva con la ayuda del software SPSS versión 22 y, de manera particular, se hizo una prueba de asociación inferencial. La estadística descriptiva permitió determinar la prevalencia de Cryptosporidium spp. y Giardia spp. tanto en los niños, el agua y en los terneros; la prevalencia en cada caso se determinó como sigue:

- en terneros y en niños, cuantos tenían presencia de ooquistes sobre el total expresado en porcentaje;

- en agua, número de ooquistes/ml.

Los resultados se presentan a través de la media $(\overline{\mathrm{X}})$ y su respectiva desviación típica $(\mathrm{DT})$. También son mencionados aquellos casos en que se advirtió la presencia simultánea de ooquistes de Cryptosporidium spp. y quistes de Giardia spp.

\section{RESULTADOS}

\subsection{Terneros}

En cuanto a Cryptosporidium spp. se determinó una prevalencia de $93.3 \%$ (112+ y 8-). En los sectores de Cachi, Calluco, Corraluco, María Auxiliadora, Pircapamba, Quimaputo, San Sebastián, Hierba Buena y Siguin la prevalencia de Cryptosporidium spp. fue de un 100\%. El sector en que se observó la prevalencia más baja fue Pallca con un 33.3\% (Tabla 1). En el caso de Giardia spp. se reportó una prevalencia de $76.7 \%$ (92+ y 28-). Los sectores que mantuvieron una prevalencia del $100 \%$ fueron: Cachi, María Auxiliadora, Pallca, Pircapamba, San Sebastián y Siguin. El sector con menor prevalencia de Giardia fue Hato de la Virgen con 44.4\% (Tabla 1). La presencia simultánea de Giardia spp. y Cryptosporidium spp. se observó en el 70\% (84+ y 36-) de los casos. Los sectores que mostraron una prevalencia simultánea en el 100\% de los casos fueron: Cachi, María Auxiliadora, Pallca, Pircapamba, San Sebastián y Siguin (Tabla 1).

\subsection{Agua}

En el caso de Cryptosporidium spp. se determinó la presencia de 5 ooquistes $/ 100 \mathrm{ml}$, mientras que de Giardia lamblia, se demostró la existencia de 10 quistes $/ 100 \mathrm{ml}$. 
Tabla 1. Prevalencia de Cryptosporidium spp., Giardia spp. o ambas simultáneamente por sectores del cantón San Fernando, en el periodo comprendido entre septiembre del 2013-abril 2014.

\begin{tabular}{lccc}
\hline \multicolumn{1}{c}{ Sector } & $\begin{array}{c}\text { Cryptosporidium } \\
\text { spp. }\end{array}$ & Giardia spp. & $\begin{array}{c}\text { Cryptosporidium spp. } \\
+ \text { Giardia spp. }\end{array}$ \\
\hline Cachi & $1.00 \pm 0.00$ & $1.00 \pm 0.00$ & $1.00 \pm 0.00$ \\
Calluco & $1.00 \pm 0.00$ & $0.83 \pm 0.41$ & $0.83 \pm 0.41$ \\
Corraluco & $1.00 \pm 0.00$ & $0.67 \pm 0.58$ & $0.67 \pm 0.58$ \\
H. Virgen & $0.94 \pm 0.24$ & $0.44 \pm 0.51$ & $0.39 \pm 0.50$ \\
M. Auxiliad & $1.00 \pm 0.00$ & $1.00 \pm 0.00$ & $1.00 \pm 0.00$ \\
Mamayacu & $0.94 \pm 0.25$ & $0.88 \pm 0.34$ & $0.81 \pm 0.40$ \\
Pallca & $0.33 \pm 0.52$ & $1.00 \pm 0.00$ & $0.33 \pm 0.52$ \\
Pircapamba & $1.00 \pm 0.00$ & $1.00 \pm 0.00$ & $1.00 \pm 0.00$ \\
Quimaputo & $1.00 \pm 0.00$ & $0.78 \pm 0.44$ & $0.78 \pm 0.44$ \\
San Sebastián & $1.00 \pm 0.00$ & $1.00 \pm 0.00$ & $1.00 \pm 0.00$ \\
Shuno & $0.93 \pm 0.26$ & $0.93 \pm 0.26$ & $0.87 \pm 0.35$ \\
Siguilla & $0.90 \pm 0.30$ & $0.57 \pm 0.51$ & $0.57 \pm 0.51$ \\
H. Buena & $1.00 \pm 0.00$ & $0.83 \pm 0.41$ & $0.83 \pm 0.41$ \\
Siguin & $1.00 \pm 0.00$ & $1.00 \pm 0.00$ & $1.00 \pm 0.00$ \\
Turupamba & $0.75 \pm 0.50$ & $0.75 \pm 0.50$ & $0.50 \pm 0.58$ \\
\hline Prevalencia media & $0.92 \pm 0.28$ & $0.77 \pm 0.42$ & $0.70 \pm 0.46$ \\
\hline
\end{tabular}

\subsection{Niños}

La prevalencia total determinada para Cryptosporidium spp. fue de $14.3 \%$ y para Giardia spp. de 33.3\%, mientras que la presencia simultánea de ambos parásitos se observó en el $4.8 \%$ de los casos. Cuando se discriminaron los casos según el origen rural o urbano de los niños muestreados, la prevalencia de Cryptosporidium spp. en los niños en la zona rural $(\mathrm{N}=25)$ alcanzó un $16.0 \%(4+$ y 21-), mientras que en la zona urbana $(\mathrm{N}=17)$ se obtuvo un $11.8 \%(2+$ y 15-). Por su parte, la prevalencia de Giardia spp. en los niños de la zona urbana alcanzó un 58.9\% (10+ y 7-), mientras que, en la zona rural fue de $16.0 \%$ (4+ y 21-) (Tabla 2). Los casos con infestación simultánea de ambos parásitos fueron de 4.0\% (1+ y 24) para la zona rural y de $5.9 \%$ para la zona urbana (1+ y 16-).

Tabla 2. Prevalencia $(\overline{\boldsymbol{X}} \pm \mathrm{DT})$ de Cryptosporidium spp., Giardia spp., y ambas simultáneamente, en niños de acuerdo a su procedencia rural o urbana del cantón San Fernando.

\begin{tabular}{ccccccccc}
\hline \multicolumn{3}{c}{ Cryptosporidium spp. } & \multicolumn{3}{c}{ Giardia spp. } & \multicolumn{3}{c}{$\begin{array}{c}\text { Cryptosporidium spp. }+ \\
\text { Giardia spp. }\end{array}$} \\
\hline Rurales & Urbanos & Totales & Rurales & Urbanos & Totales & Rurales & Urbanos & Totales \\
\hline $0.160 \pm$ & $0.12 \pm$ & $0.14 \pm$ & $0.16 \pm$ & $0.59 \pm$ & $0.33 \pm$ & $0.04 \pm$ & $0.06 \pm$ & $0.05 \pm$ \\
0.374 & 0.33 & 0.35 & 0.37 & 0.51 & 0.48 & 0.20 & 0.24 & 0.22 \\
\hline
\end{tabular}

*Chi cuadrado $\left(\mathrm{X}^{2}\right)=8.350588$, sig. 0.004, V de Cramer 0.446

\section{DISCUSIÓN}

En el presente estudio se muestran los resultados de prevalencia de dos enteroparásitos, Cryptosporidium spp. y Giardia spp., en terneros y niños, así como el nivel de presencia de estos enteroparásitos en el agua de la zona de captación para sus respectivos usos del cantón San Fernando de la provincia del Azuay en Ecuador. 


\subsection{Cryptosporidium spp. en terneros}

Se determinó que la prevalencia de este parásito osciló entre 33 y 100\% según los sectores analizados dentro del mismo cantón. La media para el mismo fue del 92\%; esto muestra un nivel de contaminación extremadamente alto. En EE.UU. se encontró una prevalencia del 50\% (Modini et al., 2010), en Argentina del 7 al 30\% (Del Coco et al., 2009), en México del 42.9\% (Cano et al., 2011), en Brasil del 27.8\% (Fitz et al., 2013), siendo valores sensiblemente inferiores a los aquí determinados. Nuestros resultados no pueden ser comparables a los obtenidos en otros países en términos estrictos ya que se trata de estudios realizados en algunas zonas de esos países y no siempre las condiciones de estos trabajos fueron similares. La zona geográfica estudiada, la edad, la historia clínica del hato o rebaño, los sistemas de explotación, las prácticas de higiene, etc., seguramente difieren en todos los casos y las mismas no son siempre descritas en detalle. De todas maneras, lo obtenido en nuestro caso delata problemas estructurales y en el caso de los animales en particular, se puede mencionar que, en la cuenca lechera del cantón San Fernando, un factor importante es que los pastizales permanecen ocupados por más tiempo y el manejo al sogueo (animales que permanecen en estaca) ayuda o favorece a la continua acumulación de ooquistes y, por ende, a la contaminación del agua y del medio ambiente.

\subsection{Cryptosporidium spp. en el agua}

Investigaciones realizadas en los Estados Unidos en 82 muestras de agua potable encontraron ooquistes de Cryptosporidium en un $26.8 \%$ con una media geométrica de 1.52 ooquistes/100L (LeChevallier et al., 1991). Sin embargo, la presencia de este protozoario no ha sido detectada en una estación de tratamiento de agua en Sao Paolo, Brasil (Henrique de Aguirre et al., 2002). Por su parte, un estudio realizado en el estado de Bolívar en Venezuela, dio a conocer una prevalencia de Cryptosporidium spp. en el $85.7 \%$ en aguas crudas (Cermeño et al., 2008). Otro estudio realizado en Venezuela, enfocado a las aguas que entran a la planta de tratamiento de aguas residuales del estado de Aragua, dio un valor promedio de 10 ooquistes/l (encontrándose dentro del rango 1-10 ooquistes). Este tipo de resultados demuestran un nivel de riesgo para infectar a animales y seres humanos, trayendo como consecuencia un alto riesgo para la salud pública, debido al uso de estas aguas para fines agrícolas y/o consumo humano (Medina et al., 2012).

Los datos citados por los diferentes autores, frente a los encontrados en la presente investigación que son de 5 ooquistes $/ 100 \mathrm{ml}$ de agua, nos indica que existe una alta prevalencia de ooquistes, siendo éste un factor determinante para la infestación de los humanos y animales aguas abajo. Se evidencia que la falta de control sanitario sobre el agua de consumo produce esta afección en los seres humanos y que los menores de edad no están exentos de esta peligrosidad.

\subsection{Cryptosporidium spp. en niños}

La prevalencia de ooquistes según encuestas realizadas en Europa está entre el 1 y 2\%, en América del Norte del 0.6 al $4.3 \%$, y en países en desarrollo entre el 10 y 20\% (Acha \& Szyfres, 2003). Un estudio realizado en el año 2007 en niños procedentes de las zonas rurales de la provincia de ChimborazoEcuador, encontraron prevalencias del 8.9\% (Jacobsen et al., 2008). Con los datos encontrados sobre éste enteroparásito en nuestro estudio, se puede encontrar mucha coincidencia con lo expuesto por Acha \& Szyfres (2003), quienes manifiestan que la prevalencia de ooquistes en países en vías de desarrollo está en promedio en el 15\%. La prevalencia de Cryptosporidium spp. encontrada en San Fernando, mostró en la zona rural 16\%, mientras que en la zona urbana alcanza un $11.8 \%$, teniendo como promedio el $14.3 \%$.

\subsection{Giardia spp. en terneros}

Beck \& Pantches (2010), refieren que en Alemania Central se ha encontrado una prevalencia de Giardia spp. del 14 al $50 \%$ en terneros de 2 y 10 semanas, en los cuales no se ha identificado relación entre la diarrea y la detección de los parásitos. En terneros de granjas lecheras de Quebec, Canadá, se reportó una prevalencia de $45.7 \%$ para este protozoario (Ruest et al., 1998). Otro estudio desarrollado en forma paralela en el oeste de Canadá y el oeste de Australia en terneros lactantes, demostró una prevalencia de 
Giardia spp. del 57 y 58\% respectivamente. Los autores de ese estudio concluyen que la infección es potencialmente zoonótica para los seres humanos de las localidades (O'Handley et al., 2000).

En Argentina se ha encontrado una prevalencia de Giardia spp. del 30.69 al $38.34 \%$ en terneros, siendo los becerros menores a las dos semanas, aquellos que tienen mayor probabilidad de ser infectados (Tiranti et al., 2011). Por su parte, un estudio desarrollado en la Sabana de Bogotá, demostró que la prevalencia de Giardia spp. en terneros asciende al 37.3\% (Hernández \& Cortés, 2012). Estos valores, confrontados con la prevalencia de 76.7\% encontrada en el cantón San Fernando, muestran que, en esta zona de producción lechera del Ecuador, la expectativa del efecto zoonótico es muy alta.

\subsection{Giardia spp. en el agua}

LeChevallier et al. (1991), encontraron que en Estados Unidos existe una prevalencia del 17.1\%, mientras que un estudio realizado en una planta de tratamiento de agua en Sao Paulo, Brasil, dio como positivo en dos de las ocho muestras de agua bruta $(40 / 100 \mathrm{ml})$, lo que equivale a un $25 \%$ (Henrique de Aguirre et al., 2002). El estudio realizado por Cermeño et al. (2008) en el estado de Bolívar en Venezuela dio a conocer una prevalencia de Giardia spp. del 71.4\% en aguas crudas. Brito (2010), en un estudio realizado en las aguas crudas que ingresan a la planta de potabilización de Sustag, CuencaEcuador, ha encontrado un equivalente a 0.01 quistes $/ 100 \mathrm{ml}$ de Giardia lamblia. En contraparte, el estudio desarrollado en el cantón San Fernando encontró una carga de 10 quistes/100 ml, ello da cuenta del riesgo de contaminación que tienen los efluentes del agua de consumo humano.

\subsection{Giardia spp. en los niños}

Los estudios realizados por Lopez Villacís (2013) en la parroquia Cunchibamba, perteneciente a la ciudad de Ambato-Ecuador, encontraron una prevalencia del 16.5\% de Giardia lamblia. El autor de dicho estudio manifiestó que se debe tomar en cuenta que la presencia de este endoparásito, reportado en su investigación, podría estar relacionada con el consumo de agua no tratada por parte de los sujetos participantes. Además, señaló que el resultado fue similar al obtenido por Serrano \& Cantilo (2001), quienes analizaron muestras de heces de 2,123 niños, de los cuales el 69.6\% presentaron protozoarios; dentro de estos, los más frecuentemente encontrados fueron E. histolytica, E. coli y Giardia lamblia.

En un estudio realizado por Sarmiento \& Román (2011) en la comunidad de Minas, perteneciente a la parroquia Baños, cantón Cuenca, encontraron una prevalencia del 19.7\%. Por su lado, Jacobsen et al. (2008), en niños rurales del Chimborazo, encontraron prevalencia del 21.1\%. En Ciudad Juárez (México) un estudio en niños, efectuado en un área con agua potable entubada, demostró que estaban parasitados con Giardia lamblia el 37.8\% de ellos; en cambio, en un área sin agua potable entubada, los niños parasitados con Giardia lamblia llegaron al 56\% (García et al., 2010). En Barranquilla, Colombia, la Giardia intestinalis está presente en el 9.7\% de las muestras analizadas, siendo el enteroparásito más prevalente (Fillot et al., 2015).

Tomando en consideración los resultados de diversas investigaciones, frente a la realizada en San Fernando con un promedio de prevalencia del $33.3 \%$, se determina que la prevalencia de Giardias spp. es alta, además, se evidencia mayor prevalencia en la zona urbana; pues ahí alcanza un 58.9\%, mientras que en la zona rural sólo es de $16.0 \%$. Con los resultados obtenidos, para la prueba de asociación ( $\mathrm{X}^{2}$ 8.351, sig. 0.004, V de Cramer 0.446), se concluye que la presencia de Giardia spp. en los niños menores de 6 años está relacionada con su procedencia, pues este parásito se presenta mayormente en aquellos casos provenientes de la zona urbana (cabecera cantonal) del cantón San Fernando. Se supone que en los niños procedentes de las zonas rurales existe mayor inmunidad y, por lo tanto, más resistencia al parásito. Es menester señalar que el agua, tanto para la zona rural como para la urbana, tiene el mismo origen (tanques de captación del Hato de la Virgen). Sin embargo, una limitación del presente estudio, es la falta de un análisis parasitológico del agua potabilizada, distribuida en el sector urbano, en comparación con la no potabilizada, distribuida en el sector rural. 


\section{CONCLUSIÓN}

De acuerdo a nuestros resultados, la prevalencia de Cryptosporidium spp. es muy alta en la zona de estudio, lo cual evidencia un problema zoonótico. Por otra parte, la especie C. parvum, cuya presencia fue encontrada en las muestras de agua analizadas, se considera de alta patogenicidad. De igual manera, la prevalencia de Giardia spp. es alta, siendo más fuerte en la zona urbana. En lo referente al agua, la presencia de la especie $G$. lamblia, determinada por el laboratorio, es considerada, al igual que el $C$. parvum, como protozoarios de alto riesgo y patogenicidad. Con las prevalencias halladas es necesario empezar a tomar medidas para contrarrestar su presencia. Los datos expuestos en este estudio presentan desafíos para todas y cada una de las instituciones gubernamentales y no gubernamentales, como gobiernos seccionales, GADs y demás personas involucradas en el quehacer diario del cantón.

\section{Bibliografía}

Acha P, Szyfres B (2003). Zoonosis y enfermedades transmisibles comunes al hombre y a los animales: Clamidiosis, rickettsiosis y virosis ( $3^{\mathrm{a}}$ ed., Vol. II). Washinton, EE.UU.: Organización Panamericana de la Salud, Oficina Sanitaria Panamericana

Agency Enviornmental Protection (2012). Cryptosporidium and Giardia in water by filtration/IMS/FA. EPA, Office of Ground Water and Drinking Water. Cincinnati, EE.UU.: AEP

Alarcón M, Beltrán M, Cárdenas M, Campos M (2005). Recuento y determinación de viabilidad de Giardia spp. y Cryptosporidium spp. en aguas potables y residuales en la cuenca alta del río Bogotá. Biomédica 25(3):353-365

Arizona Department of Environmental Quality (1995). Cryptosporidium y el agua potable. Arizona Department of Environmental Quality, Departamento de Calidad Ambiental de Arizona. Phoenix, Arizona: ADEQ

Beck W, Pantchev N (2010). Zoonosis parasitarias. España: Servet Editorial

Brito L (2010). Calidad bacteriológica y parasitaria del agua cruda de la planta de tratamiento de agua de Sustag. Cuenca, Ecuador: Facultad de Ciencias Químicas Escuela de Bioquímica y Farmacia, Universidad de Cuenca

Cano P, Alonso M, Figueroa J, Trigo F (2011). Prevalence and inicidence of Cryptosporidium spp. in calves from the Central Región of Veracruz, México. Tropical and Subtropical Agroecosystems 13(3):567-571

Carreño M, Velasco C, Rueda E (2005). Prevalencia de Cryptosporidium spp. en niños menores de 13 años con afecciones oncológicas. Colombia Médica 36(2):6-9

Cermeño J, Yori N, Arenas J, Hernández I (2008). Cryptosporidium Parvum y Giardia Lamblia en aguas crudas y tratadas del Estado Bolívar, Venezuela. Universidad, Ciencia y Tecnología 12(46):39-42

De Arango M, Rodríguez D, Prada N (2006). Frecuencia de Cryptosporidium spp. en materia fecal de niños entre un mes y trece años en un hospital local colombiano. Colombia Médica 37(2):121-125

Del Coco F, Córdova M, Basualdo J (2009). Criptosporidiosis: una zoonosis emergente. Revista Argentina de Microbiología 41(3):185-196

Fillot M, Guzmán J, Cantillo L, Gómez L, Sánchez L, Acosta B, Sarmiento-Rubiano L (2015). Prevalencia de parásitos intestinales en niños del Área Metropolitana de Barranquilla, Colombia. Revista Cubana de Medicina Tropical (Online) 67(3):1-7

Fitz E, Rosario R, Hernández R, Hernández E, Rodríguez E, García Z (2013). Cryptosporidium parvum: prevalencia y factores de riesgo en becerros del municipio de Cuajinicuilapa, Guerrero, México. Veterinaria y Zootecnia 7(1):49-61

García J, Salazar E, Orona I, Fortis M, Trejo H (2010). Agricultura Orgánica. Juárez, Méjico: Informe, Facultad de Agricultura y Zootecnia, Universidad Juárez del Estado de Durango

Giraldo J, Lora F, Henao L, Mejía S, Gómez J (2005). Prevalencia de Gardiasis y parásitos intestinales en preescolares de hogares atendidos en un programa estatal en Armenia, Colombia. Revista Salud Pública 7(3):327-338 
Henrique de Aguirre G, Bueno Franco R, de Lima lsaac R (2002). Ocurrencia de Giardia spp. y Cryptosporidium spp. en una estación de tratamiento de agua (ETACAPIM FINO), Sao Paulo, Brasil. Rev Perú Parasitol 16(1):47-50

Hernández N, Cortés J (2012). Prevalencia y factores de riesgo de Cryptosporidium spp. y Giardia spp. en terneros de ganado lechero de la zona noroccidental de la Sabana de Bogotá. Revista de Salud Pública 14(1):169-181

Jacobsen K, Ribeiro P, Quist B, Rydbech B (2008). Prevalence of intestinal parasites in young Quichua children in the highlands of rural Ecuador. Scielo 25(4):399-405

LeChevallier M, Norton W, Lee R (1991). Giardia spp. and Cryptosporidium spp. in filtered drinking water supplies. Applied and Environmental Microbiology 57(9):2617-2621

López Villacís N (2013). Influencia de la calidad de agua de consumo humano en la presencia de parasitosis intestinal en niños de 5 a 9 años de la parroquia Cunchibamba durante el periodo Marzo-Agosto 2012. Ambato (Ecuador): Tesis, UTA

Lozano A, García G, Galván A, Botero J (2009). Prevalencia de parásitos intestinales en niños que asisten al Templo Comedor Sagrado Corazón Teresa Benedicta de la Cruz, del barrio Vallejuelos, Medellín, 2007. Iatreia 22(3):227-234

Medina C, González A, Rueda M, Rojas G (2012). Detección de ooquistes de Cryptosporidium spp. en la planta de tratamiento de aguas residuales "Taiguaiguay" del Estado Aragua, Venezuela año 2011. Revista Ibero-Latinoamericana de parasitología 71(1):83-89

Modini L, Otero J, Carrera E, Zerbatto M, Eliggi S, Abramovich B (2010). Cryptosporidium spp. en ganado bovino: su potencial como contaminante de los recursos hídricos. FAVE, Ciencias Veterinarias 9(1):33-38

O’Handley RM, Olson ME, Fraser D, Adams P, Thompson RCA (2000). Prevalence and genotypic characterization of Giardia in dairy calves from Western Australia and Western Canada. Veterinary Parasitology 90(3):193-200

Ruest N, Faubert G, Couture Y (1998) Prevalence and geographical distribution of Giardia spp. and Cryptosporidium spp. in dairy farms in Quebec. Canadian Veterinary Journal 39(1):697-700

Sarmiento J, Román V (2011). Control de la calidad microbiológica del agua y determinación de la prevalecnia parasitológica intestinal en los alumnos de la Escuela Fiscal Mixta Segundo Espinoza Calle Minas-Baños. Cuenca, Ecuador: Informe, Universidad de Cuenca

Serrano E, Cantillo A (2001). Las parasitosis intestinales más frescuentes en la población infantil del área de salud de San Ramón de Alajuela. Acta Médica Costarricense 43(3):114-118

Solarte Y, Peña M, Madera C (2006). Transmisión de protozoarios patógenos a través del agua para consumo humano. CM Colombia Médica, Universidad del Valle 37(1):78-82

Tedesco R, Camacaro Y, Morales G, Amaya I, Blanco Y, Devera R (2012). Parásitos intestinales en niños de hogares de cuidado diario comunitarios de ciudad Bolívar, Estado Bolívar, Venezuela. Biomedicina 24(2): 142-150

Tiranti K, Larriestra A, Vissio C, Picco N, Alustiza F, Degioanni A, Vivas A (2011). Prevalence of Cryptosporidium spp. and Giardia spp., spatial clustering and patterns of shedding in dairy calves from Córdoba, Argentina. Revista Brasileira de Parasitologia Veterinária (Online) 20(2):140-147 